\title{
Blocking of the EGFR-STAT3 signaling pathway through afatinib treatment inhibited the intrahepatic cholangiocarcinoma
}

\author{
CHANGHE ZHANG ${ }^{1 *}$, HONG XU ${ }^{2 *}$, ZHENPING ZHOU $^{2}$, YE TIAN ${ }^{1}$, \\ XIAOFEI CAO ${ }^{1}$, GUOCHANG CHENG $^{1 *}$ and QINGHONG LIU ${ }^{1 *}$ \\ Departments of ${ }^{1}$ Hepatobiliary Surgery and ${ }^{2}$ Digestive Medicine, Taizhou People's Hospital, \\ Taizhou, Jiangsu 225300, P.R. China
}

Received October 5, 2015; Accepted November 25, 2016

DOI: $10.3892 /$ etm.2018.6038

\begin{abstract}
Epidermal growth factor receptor (EGFR) and downstream signal transducer and activator of transcription 3 (STAT3) signaling have been extensively implicated in various human neoplasms. Recently, a novel EGFR inhibitor, known as afatinib, has exhibited broad antitumor activities in a variety of tumors. Therefore, the present study attempted to investigate the impact of this agent on intrahepatic cholangiocarcinoma (ICC). Initially, immunohistochemical assays were performed on 15 human ICC specimens and their adjacent tissues in order to assess the protein levels of phosphorylated EGFR (pEGFR) and pSTAT3. Subsequently, the human ICC cell lines JCK and OZ were exposed to different doses of afatinib, and then cell viability and apoptosis were determined by MTT assay and flow cytometry, respectively. Furthermore, immunoblotting was applied to detect any variations in the phosphorylated protein levels of EGFR and STAT3 in afatinib-treated ICC cells. The results of the current study demonstrated that ICC specimens had evidently increased pEGFR and pSTAT3 protein levels as compared with the adjacent noncancerous tissues. Further in vitro experiments indicated that afatinib evidently blocked ICC cell growth and induced cell apoptosis. At the protein level, pEGFR and pSTAT3 were evidently attenuated by afatinib-administration. In conclusion, the present study clearly determined that afatinib exerts an antitumor effect on ICC cells by silencing the EGFR-STAT3 signaling pathway. This novel agent deserves further investigation as a potential therapeutic strategy for ICC.
\end{abstract}

Correspondence to: Dr Qinghong Liu, Department of Hepatobiliary Surgery, Taizhou People's Hospital, 399 Hailing Southern Road, Taizhou, Jiangsu 225300, P.R. China

E-mail: doctorlqh@163.com

*Contributed equally

Key words: afatinib, intrahepatic cholangiocarcinoma, epidermal growth factor receptor, signal transducer and activator of transcription 3

\section{Introduction}

Intrahepatic cholangiocarcinoma (ICC) represents the second most commonly diagnosed primary liver malignant tumor, accounting for $\sim 3 \%$ of all gastrointestinal malignancies globally, and both incidence and mortality rates are increasing (1). Only small proportion of patients with early-stage ICC are likely to survive in the long-term, even following radical hepatectomy; furthermore, the majority of patients are diagnosed at a later stage and have poor prognosis (2). Furthermore, all available treatment strategies for ICC, including chemotherapy and immunotherapy, induce a poor response from patients. Thereby, developing novel therapeutic agents against ICC remains highly important.

The epidermal growth factor receptor (EGFR) belongs to the ErbB receptor tyrosine kinase family. Through the binding of ligands to its extracellular ligand-binding domain, EGFR can be phosphorylated to form homodimers or heterodimers, and consequently initiates extensive intracellular signaling cascades $(3,4)$. As one of the most important downstream effectors, signal transducer and activator of transcription 3 (STAT3) can be further phosphorylated at the sites of Tyr-705 or Ser-727 by activated EGFR, and can then translocate into the nucleus to exert transcriptional regulations, primarily contributing to cell proliferation, resistance to apoptosis and angiogenesis $(5,6)$. Thus far, aberrant EGFR-STAT3 flux has been widely implicated to various neoplasms, such as colorectal, breast and lung cancer $(4,5,7)$. All these features indicate that EGFR may be an intriguing target for developing novel antitumor agents. Numerous small-molecule EGFR inhibitors known as tyrosine kinase inhibitors (TKIs) have exhibited varying anticancer effects to date $(3,8,9)$. However, due to the secondary resistance primarily induced by EGFR mutations, various TKIs have caused unsatisfactory therapeutic effects in previous clinic studies $(8,10,11)$.

A novel irreversible TKI agent known as afatinib has been identified $(10,11)$. Due to its increased inhibiting potency and multitarget suppressing ability on ErbB receptor tyrosine kinases, afatinib exhibits relative superiority over other conventional first-generation EGFR inhibitors $(8,12)$. To date, studies have demonstrated that afatinib has extensive antitumor efficacies on various neoplasms, particularly on metastatic or refractory tumors $(10,11)$. These findings further establish 
afatinib among the first-line options of EGFR-targeted therapeutic strategies, whether as monotherapy or an important compound in combined treatment. More recently, phase III clinic studies of afatinib on non-small cell lung cancer have also been conducted (8). However, the potential carcinogenic impact of EGFR-STAT3 signaling axis on ICC has not been fully validated. In addition, to the best of our knowledge, the effect of afatinib treatment on ICC has not been investigated previously. Therefore, the present study was conducted with the aim of identifying the effect of afatinib treatment on ICC.

\section{Materials and methods}

Cells and reagents. Human ICC cell lines OZ and JCK were purchased from the Chinese Academy of Sciences (Shanghai, China). Afatinib (cat. no. SC-364398) was obtained from Santa Cruz Biotechnology, Inc. (Santa Cruz, CA, USA). Dulbecco's modified Eagle's medium, fetal bovine serum, MTT cell proliferation and cytotoxicity assay (cat. no. C0009) and BCA protein assay (cat. no. P0012S) kits were all obtained from Beyotime Institute of Biotechnology (Nanjing, China). The Apo-BrdU-Red In Situ DNA Fragmentation Assay kit (cat. no. K404-60) was from BioVision, Inc. (Tucson, AZ, USA). Primary rabbit monoclonal antibodies against phosphorylated EGFR (pEGFR; cat. no. ab40815), pSTAT3 (cat. no. ab76315) and GAPDH (cat. no. 8227), as well as the horseradish peroxidase-conjugated goat anti-rabbit IgG secondary antibody (cat. no. 97080) were all purchased from Abcam (Cambridge, MA, USA).

Immunohistochemical analysis. In order to determine the protein levels of phosphorylated EGFR and STAT3, 15 ICC specimens ( $\mathrm{T}$ group) and matched adjacent normal tissues (N group) were retrieved from the Department of Pathology for immunohistochemistry assessment. Written informed consent was obtained from all participants. The participants constituted of 9 male and 6 female patients with a mean age of 53.5 years old. No patients received chemotherapy prior to anatomic heptectomy, which was performed between July 2013 and October 2014. ICC specimens which, were $20 \mu \mathrm{m}$ thick were then fixed in $10 \%$ formaldehyde at $4^{\circ} \mathrm{C}$ for $24 \mathrm{~h}$ prior to paraffin embedding. All cases were approved by the Ethical Review Committee (Institutional Ethical Board of Taizhou People's Hospital, Taizhou, China) and conformed closely to the Declaration of Helsinki.

Consecutive sections $(4 \mu \mathrm{m})$ of the paraffin-embedded normal and tumor specimens were prepared. The protein levels of phosphorylated EGFR and STAT3 in these sections were detected using antibodies against pEGFR (1:100) and pSTAT3 (1:150), respectively. Two individual pathologists blinded to the groups scored these sections using an Olympus CX32 microscope (Olympus Corp., Tokyo, Japan). The protein expression extent was scored into four gradients according to the staining intensity, as follows: 0 , absence of staining $(<9 \%$ staining); 1, mild expression (10-19\% staining); 2, moderate expression (20-49\% staining); and 3, high expression ( $>49 \%$ staining). Subsequently, the mean percentage of positive tumor cells from at least five different fields-of-view at magnification of $\mathrm{x} 400$ was calculated for each sample. The percentages of positive tumor cells and the staining intensity were multiplied to produce a weighted score for each case, with the total scores ranging between $0(<10 \%)$ to $3(>49 \%)$.

Cell preparation and viability measurement. To determine the inhibitory effect of afatinib on ICC, the human ICC cell lines JCK and OZ were incubated in 96-well plates (Nunc; Thermo Fisher Scientific, Inc., Waltham, MA, USA) at a density of $5 \times 10^{3}$ cells/well and cultured in Dulbecco's modified Eagle's medium supplemented with $10 \%$ fetal bovine serum at $37^{\circ} \mathrm{C}$ for $4 \mathrm{~h}$. Subsequently, cells were exposed to afatinib at concentrations of 1, 10, 50, 100 and $150 \mathrm{nM}$, respectively. Cells without afatinib treatment were used as the control (ctrl) group. When cells in the ctrl group had almost reached confluence, $20 \mu \mathrm{l}$ MTT $(5 \mathrm{mg} / \mathrm{ml})$ was added to each well. The ICC cells were then incubated at $37^{\circ} \mathrm{C}$ for a further $4 \mathrm{~h}$. Subsequently, the supernatant was removed and $150 \mu 10.1 \%$ dimethyl sulfoxide (vehicle) was added to every well to resolve the MTT. The absorbance of each well was ultimately measured at $450 \mathrm{~nm}$ on a Multiskan photometer (Thermo Fisher Scientific, Inc.). Subsequently, the half maximal inhibitory concentration $\left(\mathrm{IC}_{50}\right.$ ) values of afatinib in the JCK and $\mathrm{OZ}$ cell lines were calculated by statistical analysis, and found to be 54.6 and $35.2 \mathrm{nM}$, respectively. Furthermore, the growth responses of the two cell lines to an intermediate concentration of afatinib (30 $\mathrm{nM})$ at different time points $(0,24,48$ and $72 \mathrm{~h})$ were determined in order to determine the optimum incubation time.

For subsequent apoptosis measurement and protein detection experiments, the ICC cell lines were cultured in 6-well plates (Nunc; Thermo Fisher Scientific, Inc.) with $30 \mathrm{nM}$ afatinib and harvested after $48 \mathrm{~h}$ incubation.

Apoptosis detection. To evaluate the apoptosis variances in ICC cell lines following afatinib exposure, the commercial Apo-BrdU-Red In Situ DNA Fragmentation assay kit (BioVision, Inc., Milpitas, CA, USA) was employed. The BrdUTP in this TUNEL-staining kit was able to actively bind DNA strand breaks, which were then identified by a red fluorescence labeled anti-BrdU monoclonal antibody, and read by flow cytometry (Excitation/Emittance wavelengths, 488/576 nm). For sample preparation, ICC cells were collected following afatinib treatment, resuspended into phosphate-buffered saline containing $1 \%(\mathrm{w} / \mathrm{v})$ formaldehyde and stored at $4^{\circ} \mathrm{C}$. Fixed cells were washed with precooling wash buffer twice at $4^{\circ} \mathrm{C}$. Cells were incubated in the DNA Labeling Solution for $60 \mathrm{~min}$ at $37^{\circ} \mathrm{C}$, then Rinse Buffer were added and the sample was centrifuged for $5 \mathrm{~min}$. Cells were subsequently incubated with antibody solution in the dark for $30 \mathrm{~min}$ at room temperature. A total of $0.5 \mathrm{ml}$ of propidium iodide/RNase A solution was added and the sample was incubated in the dark for $30 \mathrm{~min}$ at room temperature. Flow cytometry was performed using a Beckman Coulter Epics XL instrument (Beckman Coulter, Inc., Brea, CA, USA) and analyzed using EXPO32TM ADC software (Beckman Coulter, Inc.). All above mentioned buffers were part of the Apo-BrdU-Red In Situ DNA Fragmentation assay kit (BioVision, Inc.). Further procedures were performed according to the manufacturer's instructions.

Western blotting. Cell samples grown in 6-well plates were incubated with ice-cold lysis buffer containing $0.1 \%$ Triton X-100, 50 mM HEPES (pH 7.5), 150 mM NaCl, 10\% 
A

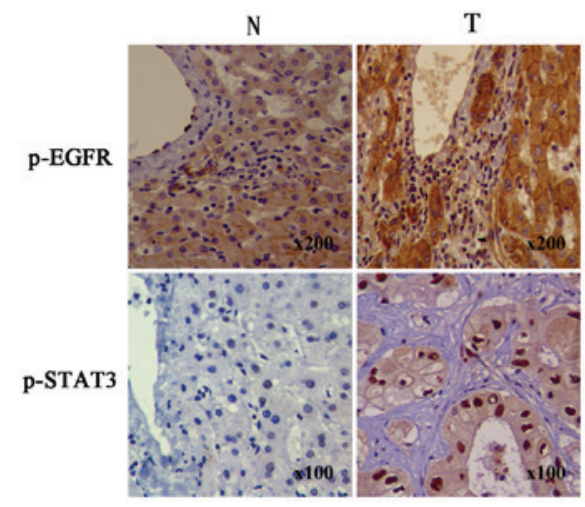

B

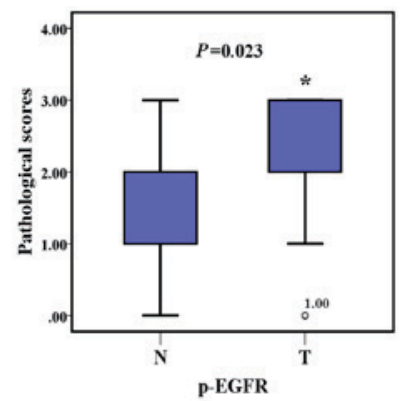

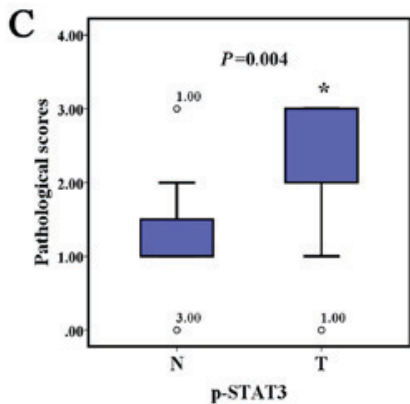

Figure 1. ICC tissues displayed significant protein expression of pEGFR and pSTAT3. (A) Immunohistochemical images showing the pEGFR and STAT3 content of $\mathrm{N}$ and $\mathrm{T}$ tissues. (B) pEGFR and (C) pSTAT3 expression levels in the ICC specimens were evidently higher compared with those of adjacent noncancerous liver tissues $\left(\mathrm{n}=3 ;{ }^{*} \mathrm{P}<0.05\right)$. ICC, intrahepatic cholangiocarcinoma; pEGFR, phosphorylated epidermal growth factor receptor; pSTAT3, phosphorylated signal transducer and activator of transcription 3; o, represent outliers; N, normal; T, tumor.

(v/v) glycerol, $1.5 \mathrm{mM} \mathrm{MgCl}_{2}, 1 \mathrm{mM}$ dithiothreitol, $1 \mathrm{mM}$ sodium fluoride, $0.1 \mathrm{mM}$ sodium orthovanadate, $1 \mathrm{mM}$ phenylmethylsulfonyl fluoride, $2 \mathrm{mg} / \mathrm{ml}$ leupeptin and $2 \mathrm{mg} / \mathrm{ml}$ aprotin in. Total protein was centrifuged at $12,000 \mathrm{x}$ g at $4^{\circ} \mathrm{C}$ for $10 \mathrm{~min}$, and the supernatants were subjected to bolting at $100^{\circ} \mathrm{C}$ by iron heating for a further $10 \mathrm{~min}$ to degrade protein. The protein concentration of the sample was determined by a BCA protein assay kit, and equivalent protein samples $(30 \mathrm{mg}$ ) were then subjected to $12 \%$ SDS-PAGE. Subsequently, the proteins were transferred to nitrocellulose membranes and probed with specific primary antibodies against pEGFR $(1: 1,500)$, pSTAT3 $(1: 2,000)$ and GAPDH $(1: 2,000)$ at $4{ }^{\circ} \mathrm{C}$ for $12 \mathrm{~h}$. Next, the samples were incubated with the secondary antibody $(1: 2,000)$ at room temperature for $1 \mathrm{~h}$. The molecular sizes of the targeted proteins were determined by comparison with prestained Precision Plus Protein ${ }^{\mathrm{TM}}$ Dual Xtra protein markers (Bio-Rad Laboratories, Inc., Hercules, CA, USA). The protein bands were then scanned using the Western Lightning Chemiluminescent Reagent Plus (PerkinElmer, Inc., Boston, MA, USA) detection system. Protein immunoreactivity was subsequently detected by the Gel Doc XR system (170-8170) and analyzed using Image J software (https://imagej.nih. gov/ij/docs/index.html). GAPDH served as the loading control in the western blotting experiments.

Statistical analysis. All parametric data are expressed as the mean \pm standard error, and were analyzed by Student's t-test. Non-parametric data analysis was performed by Mann-Whitney test. For all tests, analyses were conducted using the SPSS version 19.0 statistical software (IBM SPSS, Armonk, NY,
USA) and a two-sided $\mathrm{P}<0.05$ was considered to indicate statistically significant differences.

\section{Results}

ICC specimens demonstrate increased pEGFR and pSTAT3 protein expression levels compared with normal tissues. In order to investigate the pEGFR and downstream pSTAT3 protein variance between human ICC and normal liver tissues, immunohistochemical assays were performed. As shown in Fig. 1, ICC specimens both presented notably increased pEGFR and pSTAT3 protein expression levels representing a higher pathological score, compared with the adjacent noncancerous tissues.

Afatinib evidently induces growth arrest in ICC cell lines. To verify the antitumor activity of afatinib, the $\mathrm{IC}_{50}$ values of afatinib treatment on the ICC cell lines JCK and OZ were determined by MTT assay. Following afatinib-pretreatment at concentrations of $1,10,50,100$ and $150 \mathrm{nM}$, the JCK cell viability rates were $95 \pm 8,68 \pm 9,51 \pm 6,44 \pm 8$ and $39 \pm 5 \%$, respectively. Similarly, the corresponding viability values in the $\mathrm{OZ}$ cell lines were $91 \pm 10$, $65 \pm 8,40 \pm 7$ and $35 \pm 6 \%$ respectively (Fig. $2 \mathrm{~A}$ ).

Following statistical analysis, the $\mathrm{IC}_{50}$ values of afatinib in control JCK and OZ cells were found to be 54.6 and $35.2 \mathrm{nM}$, respectively. Meanwhile, the JCK cell viability OD values at the time points of 24,48 and $72 \mathrm{~h}$ after $30 \mathrm{nM}$ afatinib exposure were $0.81 \pm 0.02,0.54 \pm 0.11$ and $0.23 \pm 0.04$, respectively, which were significantly lower than the corresponding values in the ctrl group $(1.01 \pm 0.04,1.73 \pm 0.11$ and $1.93 \pm 0.14$; Fig. $2 B)$. Consistently, the OZ cell survival ratios at 24,48 and $72 \mathrm{~h}$ in 
A

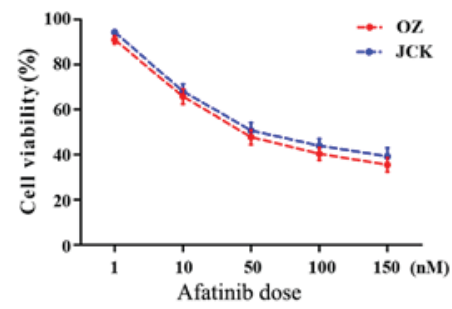

C
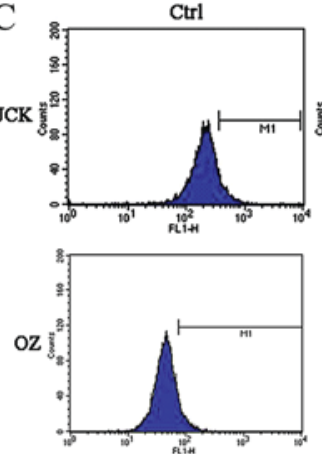

E

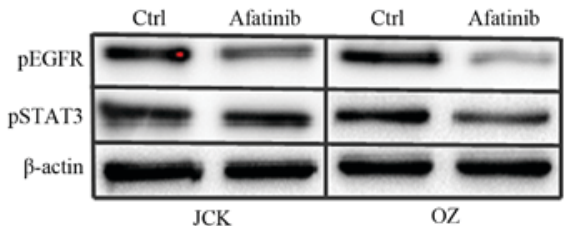

B

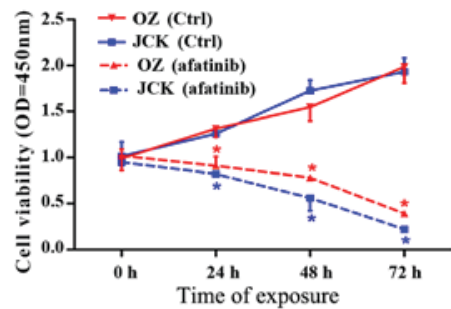

$\mathrm{D}$
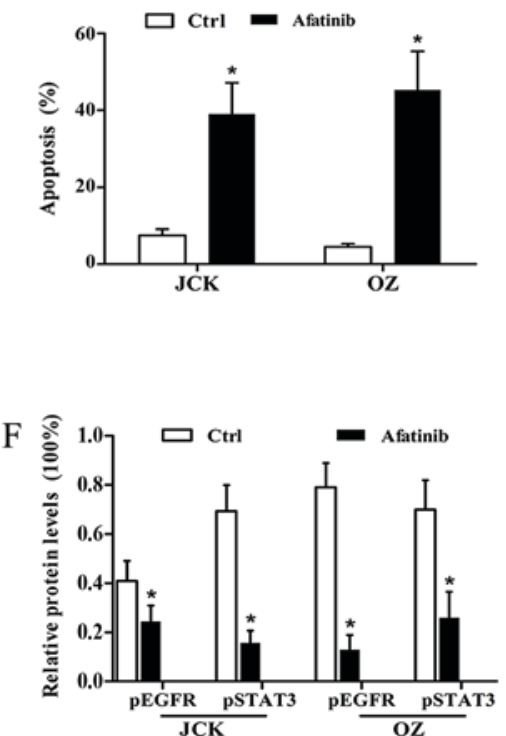

Figure 2. Afatinib induced cell apoptosis and proliferation arrest in ICC cell lines. Cell viability in JCK and OZ cells treated with (A) different afatinib doses and (B) for different durations, showing that afatinib blocked the cell growth in a time- and dose-dependent manner. (C) Flow cytometry using TUNEL-staining, and (D) cell apoptosis results, demonstrating that afatinib notably induced ICC cell apoptosis. (E) Western blots and (F) quantified protein levels, revealing that afatinib significantly inhibited pEGFR and pSTAT3 $(\mathrm{n}=3)$. ${ }^{*} \mathrm{P}<0.05$ vs. ctrl. ICC, intrahepatic cholangiocarcinoma; ctrl, control; pEGFR, phosphorylated epidermal growth factor receptor; pSTAT3, phosphorylated signal transducer and activator of transcription 3.

the afatinib group were $0.91 \pm 0.10,0.77 \pm 0.04$ and $0.36 \pm 0.06$ respectively, which were all lower compared with the associated rates in the control group $(1.31 \pm 0.09,1.55 \pm 0.15$ and $1.99 \pm 0.18$; Fig. 2B). These results demonstrated that afatinib treatment had a strong antiproliferative impact on the ICC cell lines.

Afatinib evidently promotes ICC cell apoptosis. The apoptosis response of ICC cells to afatinib treatment was detected by flow cytometry assay. After $48 \mathrm{~h}$ of exposure to $30 \mathrm{nM}$ afatinib, the cell apoptosis ratios in the JCK and OZ cell lines were $0.39 \pm 0.08$ and $0.46 \pm 0.11$ respectively, which were both markedly higher compared with the rates observed in the control groups $(0.07 \pm 0.02$ and $0.05 \pm 0.01$, respectively; Fig. $2 \mathrm{C}$ and D). These results indicated a marked pro-apoptotic effect of afatinib on ICC cells.

Afatinib significantly inhibits protein levels of $p E G F R$ and pSTAT3. To determine the variation in the protein expression levels of pEGFR and pSTAT3 in the two ICC cell lines following afatinib pretreatment, immunoblotting assays were performed (Fig. 2E and F). At the protein level, the relative pEGFR contents in afatinib-treated JCK and OZ cell were $0.24 \pm 0.07$ and $0.13 \pm 0.06$, which were both significantly reduced $(\mathrm{P}<0.05)$ compared with those in the corresponding control groups $(0.41 \pm 0.08$ and $0.79 \pm 0.10$, respectively). Relative pSTAT3 protein levels in the JCK and OZ cell lines following afatinib exposure were $0.16 \pm 0.05$ and $0.26 \pm 0.11$, respectively, which were significantly lower compared with the corresponding control group levels $(0.69 \pm 0.11$ and $0.70 \pm 0.12$, respectively; all $\mathrm{P}<0.05$; Fig. 2F). These data revealed the evident suppressing impact of afatinib on ICC cell lines.

\section{Discussion}

ICC remains one of the most challenging tumors for clinicians to deal with, due to inadequate therapeutic strategies. For the successful treatment of patients, appropriate hepatectomy plus systematic lymphadenectomy is the gold standard; however, the majority of patients cannot receive these treatments due to delayed initial presentation $(2,13)$. In addition, patients receiving radical resection may still encounter unclear distal outcomes, and the 5-year survival rate ranges between 11.2 and $23.6 \%(2,14)$. Therefore, an insight into the oncogenesis of ICC is required to facilitate the development of suitable diagnostic biomarkers, as well as antitumor drugs, and is essential for a more effective therapeutic protocol of ICC. In present study, the levels of phosphorylated EGFR were initially investigated. Despite the small samples size involved in the present study, the immunohistochemistry assay conducted demonstrated that EGFR is commonly overexpressed in ICC. This result reinforced previous suggestions that EGFR participates in ICC tumorigenesis $(13,15,16)$. Furthermore, the presence of 
overactivated EGFR has been further associated with poor long-term survival, implying the significance of conventional postoperative immunohistochemistry for ICC patients in selecting appropriate therapeutic schedules subsequent to radical resection, through the assessment of the pEGFR levels (15-17). Since EGFR can phosphorylate STAT3 at multiple sites, such as Tyr-705 and Ser-727, to form dimers, it is not surprising that enhanced pSTAT3 expression was observed in the present ICC specimens (3). Notably, the immunohistochemical assay demonstrated the expression of STAT3 in nucleus was higher than in the cytoplasm. This finding was consistent with conventional concepts that STAT3 mainly exerts transcriptional regulation by translocating into the nucleus and binding to specific promoter sequences $(18,19)$. To a certain extent, it may also support the novel viewpoint that STAT3 can also be translocated into the mitochondria and participate in the regulation of aerobic respiration and apoptosis (20).

Cancer essentially represents a consequence of imbalance between cell death and proliferation. In this sense, apoptosis is a natural defense mechanism to eliminate unhealthy cells and the loss of apoptosis has thereby been a widely accepted hallmark of cancer (21). To date, accumulating evidence has revealed that aberrant STAT3 expression can contribute to the formation of various tumors via multiple mechanisms, such as promotion of proliferation, suppression of apoptosis and induction of cancer stem cell renewal $(5,6)$. In the current study, silencing EGFR via afatinib treatment significantly promoted cell apoptosis and induced proliferation arrest in the ICC cell lines, suggesting the important effect of EGFR-STAT3 signaling on ICC formation. This result coincided closely with the findings of previous studies on other tumors, and also indicated that TKIs may be promising anticancer agents against $\operatorname{ICC}(8,12,22)$. Besides downstream STAT3, EGFR itself can also regulate various genes that operate in tumor cells through multiple interlinked signaling pathways, such as RAS-ERK-MAPK and PI3K-AKT-mTOR $(3,4)$. The current study primarily focused on investigating the STAT3 variance. However, according to the conclusions of previous studies, it can be speculated that downstream oncogenic regulators other than STAT3 may also contribute to the blocking effects of afatinib on ICC cell lines $(3,6)$.

The definite tumorigenesis role of EGFR resulted in endeavors to develop numerous EGFR targeting agents consisting of TKIs and monoclonal antibodies. However, acquired resistance remains an important challenge for clinicians when providing early EGFR targeting therapies $(8,10,11)$. To date, EGFR mutations in 790M and/or S492R sites are known as the most important contributors accounting for TKI resistance (3). To combat this problem, the novel quinazoline derivative afatinib has been proven to inhibit EGFR via irreversible covalent modification of Cys797 in the ATP binding cleft of EGFR and has displayed considerable superiority to conventional TKIs (3). Besides EGFR mutation, the upregulation of human epidermal growth factor receptor 2 (HER2), a member of the ErbB receptor tyrosine kinase family, represents another key cause of TKI resistance $(3,15)$. To date, increasing evidence has generally validated the oncogenic impact of overactivated HER2 on a variety of tumors, including breast cancer and non-small cell lung cancer $(23,24)$. Therefore, afatinib functions as a multitarget TKI by simultaneously suppressing EGFR and HER2. According to previous studies, ICC tissues are less likely than EGFR to overexpress HER $2(13,15,16)$. However, this conclusion is fundamentally based on immunohistochemical arrays on postoperative ICC specimens without using EGFR-targeted therapies. Thus far, to the best of our knowledge, no studies have been conducted to examine the anticancer effect of TKIs on ICC. The current study was therefore the first to investigate the antitumor effect of afatinib.

In conclusion, immunohistochemical assays of human ICC specimens were performed in the present study in order to assess the protein expression levels of pEGFR and pSTAT3. Subsequently, the ICC cell growth variances and protein levels of pEGFR and pSTAT3 were also detected in the present study. The findings revealed that overactivated EGFR-STAT3 signaling was closely associated to ICC formation, while silencing this pathway through afatinib treatment significantly induced ICC cell apoptosis and growth arrest. These results suggest that EGFR-targeting therapies may be promising against ICC and require further investigation.

\section{Acknowledgements}

Not applicable.

\section{Funding}

The present research was supported by a grant from the High Level Talent Project of 'Top Six Talents' in Jiangsu Province (grant no. 2016-WSN-291).

\section{Authors' contributions}

CHZ and HX contributed to the study design, intellectual content, literature research, experimental studies, data acquisition, data analysis, statistical analysis and manuscript preparation. ZPZ, YT, XFC, GCC and QHL also contributed to the literature research, study design and data analysis. $\mathrm{QHL}$ contributed to grant acquisition for this study.

\section{Ethics approval and consent to participate}

The present study was approved by the Ethics Committee of the Fifth Affiliated Hospital of Nantong University and experiments were performed in accordance with the approved guidelines and regulations. Preoperative informed consent was obtained from all patients.

\section{Availability of data and materials}

All data generated or analyzed during this study are included in this published article.

\section{Consent for publication}

Not applicable.

\section{Conflicts of interests}

The authors declare that they have no competing interests. 


\section{References}

1. Shishir KM, Gamblin TC, Kamel I, Corona-Villalobos CP, Thomas M and Pawlik TM: Multidisciplinary approaches to intrahepatic cholangiocarcinoma. Cancer 119: 3929-3942, 2013.

2. Sriputtha S, Khuntikeo N, Promthet S and Kamsa-Ard S: Survival rate of intrahepatic cholangiocarcinoma patients after surgical treatment in Thailand. Asian Pacific J Cancer Prev 14: 1107-1110, 2013

3. Robert R Jr: ErbB/HER protein-tyrosine kinases: Structures and small molecule inhibitors. Pharmacol Res 87: 42-59, 2014.

4. Bi WW, Zhang WH, Yin GH, Luo H, Wang SQ, Wang H, Li C, Yan WQ and Nie DZ: Analysis of indoleamine 2-3 dioxygenase (IDO) and EGFR co-expression in breast cancer tissue by immunohistochemistry. Asian Pac J Cancer Prev 15: 5535-5538, 2014.

5. Zhao X, Sun X and Li XL: Expression and clinical significance of STAT3, p-STAT3, and VEGF-C in small cell lung cancer. Asian Pac J Cancer Prev 13: 2873-2877, 2012.

6. Fang B: Genetic interactions of STAT3 and anticancer drug development. Cancers (Basel) 6: 494-525, 2014.

7. Ung N, Putoczki TL, Stylli SS, Ng I, Mariadason JM, Chan TA, Zhu HJ and Luwor RB: Anti-EGFR therapeutic efficacy correlates directly with inhibition of STAT3 activity. Cancer Biol Ther 15: 623-632, 2014.

8. Chong CR and Jänne PA: The quest to overcome resistance to EGFR-targeted therapies in cancer. Nat Med 19: 1389-1400, 2013.

9. Ioannou N, Seddon AM, Dalgleish A, Mackintosh D and Modjtahedi H: Treatment with a combination of the ErbB (HER) family blocker afatinib and the IGF-IR inhibitor, NVP-AEW541 induces synergistic growth inhibition of human pancreatic cancer cells. BMC Cancer 13: 41, 2013.

10. Seiwert TY, Fayette J, Cupissol D, Del Campo JM, Clement PM, Hitt R, Degardin M, Zhang W, Blackman A, Ehrnrooth E and Cohen EE: A randomized, phase II study of afatinib versus cetuximab in metastatic or recurrent squamous cell carcinoma of the head and neck. Ann Oncol 25: 1813-1820, 2014.

11. Lai WY, Chen CY, Yang SC, Wu JY, Chang CJ, Yang PC and Peck K: Overcoming EGFR T790M-based tyrosine kinase inhibitor resistance with an allele-specific DNAzyme. Mol Ther Nucleic Acids 3: e150, 2014.

12. Machiels JP, Licitra LF, Haddad RI, Tahara M and Cohen EE: Rationale and design of LUX-Head \& Neck 1: A randomised, Phase III trial of afatinib versus methotrexate in patients with recurrent and/or metastatic head and neck squamous cell carcinoma who progressed after platinum-based therapy. BMC Cancer 14: 473, 2014.
13. Harder J, Waiz O, Otto F, Geissler M, Olschewski M, Weinhold B, Blum HE, Schmitt-Graeff A and Opitz OG: EGFR and HER 2 expression in advanced biliary tract cancer. World J Gastroenterol 15: 4511-4517, 2009.

14. Shen WF, Zhong W, Xu F, Kan T, Geng L, Xie F, Sui CJ and Yang JM: Clinicopathological and prognostic analysis of 429 patients with intrahepatic cholangiocarcinoma. World J Gastroenterol 15: 5976-5982, 2009.

15. Yoshikawa D, Ojima H, Iwasaki M, Hiraoka N, Kosuge T, Kasai S, Hirohashi S and Shibata T: Clinicopathological and prognostic significance of EGFR, VEGF, and HER2 expression in cholangiocarcinoma. Br J Cancer 98: 418-425, 2008.

16. Clapéron A, Mergey M, Aoudjehane L, Ho-Bouldoires TH, Wendum D, Prignon A, Merabtene F, Firrincieli D, DesboisMouthon C, Scatton O, et al: Hepatic myofibroblasts promote the progression of human cholangiocarcinoma through activation of epidermal growth factor receptor. Hepatology 58: 2001-2011, 2013.

17. Nanda S: Cancer: A limited role for dual EGFR and ErbB2 inhibition in cholangiocarcinoma? Nat Rev Gastroenterol Hepatol 7: 591, 2010.

18. Wu J, Patmore DM, Jousma E, Eaves DW, Breving K, Patel AV, Schwartz EB, Fuchs JR, Cripe TP, Stemmer-Rachamimov AO and Ratner N: EGFR-STAT3 signaling promotes formation of malignant peripheral nerve sheath tumors. Oncogene 33: 173-180, 2014.

19. Wendt MK, Balanis N, Carlin CR and Schiemann WP: STAT3 and epithelial-mesenchymal transitions in carcinomas. JAKSTAT 3: e28975, 2014

20. Qi QR and Yang ZM: Regulation and function of signal transducer and activator of transcription 3. World J Biol Chem 5: 231-239, 2014.

21. Mobahat M, Narendran A and Riabowol K: Survivin as a preferential target for cancer therapy. Int J Mol Sci 15: 2495-2516, 2014.

22. Concha-Benavente F, Srivastava RM, Ferrone S and Ferris RL: EGFR-mediated tumor immunoescape: The imbalance between phosphorylated STAT1 and phosphorylated STAT3. Oncoimmunology 2: e27215, 2013.

23. Zhou J, Liu Y, Wang T, Zhang H, Du M, Zhang S, Wu S, Song S, Liu B, Zhang H and Jiang Z: Serum HER2 ECD level and its clinical significance in advanced breast cancer patients with different molecular subtypes. Zhonghua Yi Xue Za Zhi 94: 1384-1387, 2014 (In Chinese).

24. Parums DV: Current status of targeted therapy in non-small cell lung cancer. Drugs Today (Barc) 50: 503-525, 2014. 